\title{
Estimation and Calculation Procedures of the Technical Provisions for Outstanding Insurance Claims
}

\author{
Aleksandrs Matvejevs, Riga Technical University, Anatoliy Malyarenko, Mälardalen University, Andrejs Matvejevs, Riga \\ Technical University
}

\begin{abstract}
The paper presents algorithms for insurance technical provisions taking into account losses, which are incurred but not reported. Evaluation of insurance technical provisions for the kinds of insurance, such as Motor Third Party Liability (MTPL) Insurance, Property Insurance and some others, have difficulties in assessing the impact of the losses from insurance claims incurred requiring a longer time for the settlement of insurance claims. These insurance requirements are mainly associated with health insurance in the MTPL Insurance, losses related to compensation for moral injuries, as well as on life care and life-long pension. To run these payments, you need to know the financial indicators for the period of settlement of loss (such as the effective interest rate, investment income, etc.) In the article the procedures for the most accurate forecast possible losses for the expected excess of loss amount for a treaty year are provided, using the loss experience of the previous years of the occurrence with their development. However, certain adjustments should be made to take account of the impact of losses from previous years for the current period. This article describes how outstanding losses have to be projected on a year of reporting, so that they are correspond to the current values.
\end{abstract}

Keywords - Excess of loss, insurance technical provisions, motor vehicle liability insurance, outstanding insurance claims, loss burden triangle.

\section{INTRODUCTION}

The problem of determining factors for prediction of outstanding claims and creating suitable technical provisions to meet these requirements is an important part of the business of insurance and Motor Third Party Liability. In fact, the published profit of these companies depends not only on the actual insurance payments, but also on the claims forecasts, which will be payable. It is very important therefore to understand correctly access a reliable assessments provisions to be set aside to cover claims, in order to ensure the financial stability of the company in calculating its profits and losses. There is a number of ways that have proved useful in practice; one of well-known form is the method of chain ladders technique. Statistical algorithms for the analysis of these data, built in recent years, include the methods of valuation, and methods of generalized linear models. The aim of this paper is to consolidate these results in order to show how the technology of the chain ladder method can be improved and expanded without changing the fundamental basis on which it was built before these changes. Two problems with the technique of chain ladder method can be considered. First of all, not enough connection between the accident years, therefore model has many parameters and volatile forecast. Secondly, the scheme development requirements is the same for all accident year. The chain ladder method does not accept any changes in the speed, at which claims are settled, or for other factors which may change the shape of the run-off triangle development. Before describing the methods to overcome these problems, first define the chain ladder method linear model and show how it can be used to give upper prediction bounds for the total amount of the outstanding claims.

\section{DATA REQUIREMENTS}

\section{A. Claims Loss Data}

As a starting point for using run-off triangle techniques to estimate future claims losses from existing insurance policies, an insurance undertaking requires information regarding its past claims experience. Specifically, it will need data for the total amounts of claims that were settled in the past. These claims losses include:

a) The claims benefit payments (gross of reinsurance recoveries).

b) Any indirect expenses that were allocated to the claims.

c) Any direct claims handling expenses.

d) The reinsurance recoveries (or other recoveries).

Reinsurance recoveries result in a reduction in claims losses (or no change). In order to simplify the explanations, these potential negative claims losses are ignored in this paper. In order to represent the claims loss data in run-off triangles the following information is required for each individual claim loss settlement, see [1]:

a) The claim event in respect of which the claims loss is settled.

b) The date of the claim event (e.g. the date on which storm damage was caused to an insured property).

c) The date on which the claim event was reported to the insurer.

d) The date on which the claim loss was settled. The benefits and the expenses in respect of a claim may be paid or allocated over a number of reporting periods. 
Using all of the above data allows an insurer to model delays between the occurrence of the claim event and the reporting of the claim event (reporting delay, used in provisioning for "incurred but not reported claims") and the delays between the reporting and the settlement of the claim (settlement delays). For simplicity purposes, this paper considers only the settlement delays, i.e., only the reported claims are considered [1], [2].

However, the approach for determining reporting delays for incurred but not reported claims is similar.

\section{MATHEMATICAL MODEL}

\section{A. Basic Terminology and Definitions}

\section{Calendar year}

A period of 12 months running from January to December.

\section{Claims occurrence year, $y$}

The calendar year in which a claim event occurred; $y \in\{1900 ; 1901 ; \ldots ; Y\}$, where $Y$ represents the most recent complete calendar year. Also called: accident year.

\section{Development year, $k$}

The complete number of years that have elapsed between the end of the claims occurrence year and the end of the year in which a claims loss amount is settled (partly or in full); $k \in\{0 ; 1 ; \ldots ; n\}$ where $n$ represents the maximum number of development years observed for any claims occurrence year.

\section{Incremental claims loss settled, $Z(y ; k)$}

The claims loss amount, for claims occurrence year $y$, that is settled in development year $k$.

It should be clear that $y+k$ represents the calendar year in which the incremental claims loss settlement amount is paid. The incremental claims loss settlement information for the claims occurrence years $Y-n$ to $Y$ can be represented as in Fig. 1 [2].

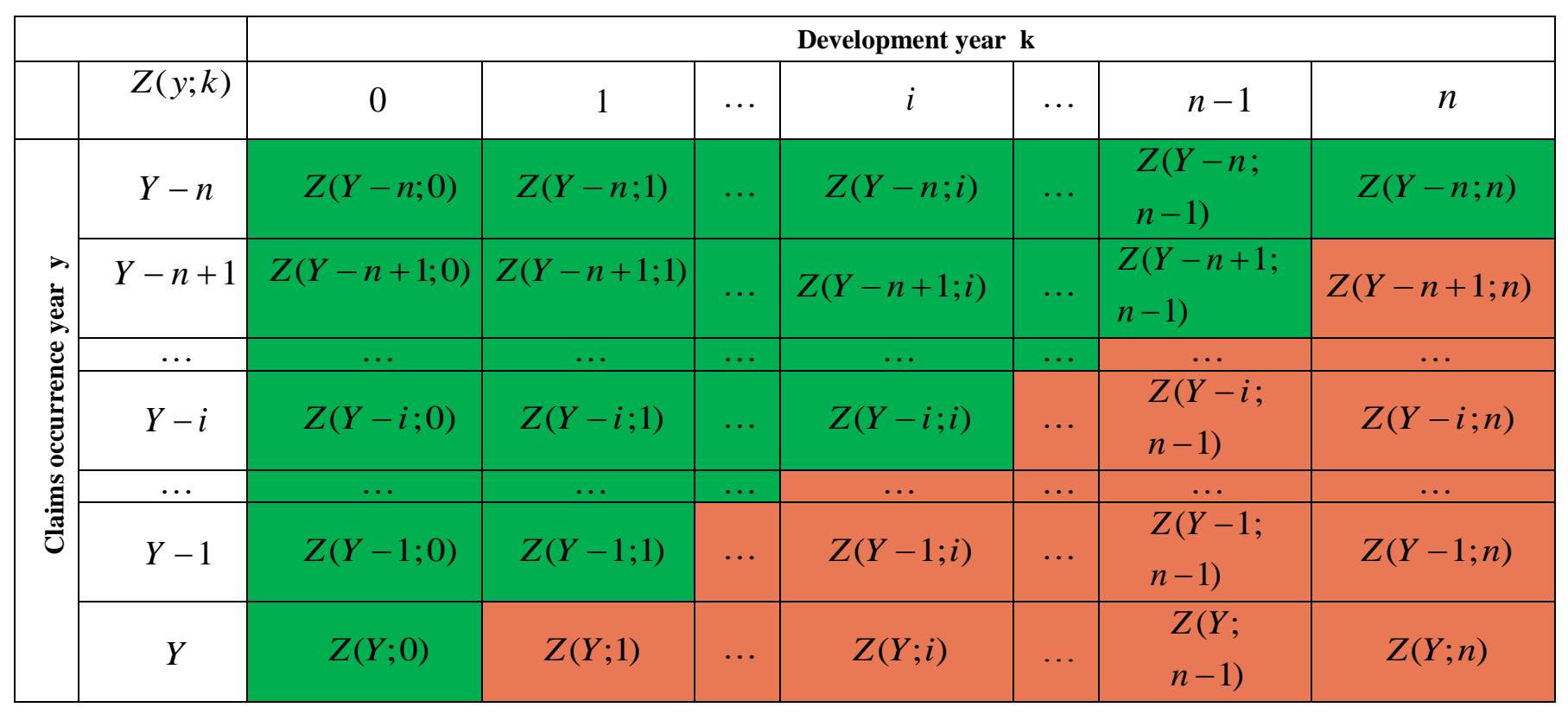

Fig. 1. Incremental claims loss settlement data represented as a run-off triangle.

In any cell in Fig. $1 Z(y ; k)$ represents the claims loss amount settled in calendar year $y+k$ in respect of claims events which occurred in year y. For example, the amount represented by the value $Z(Y-n+1 ; 1)$ was settled during the calendar year $Y-n+2$. It should be clear that in each green cell on the diagonal blue line $y+k$ always equals $Y$. This diagonal, therefore, represents the amounts settled in the most recent completed calendar year, $Y$. The upper triangle (green cells) represents settlement payments made before the end of calendar year $Y$, i.e. in the past (observed data). The lower triangle (red cells) represents the settlement payments that will be made in the future, i.e. after the most recent calendar year, $Y$. The red cells represent the settlement payments which must be estimated or predicted.
Based on the definition of $n$, claims occurrence year $Y-n$ is the only year for which claims are (expected to be) fully settled. For all other claims occurrence years it is expected that there will be further claims settlements in the future [7], [8].

\section{Cumulative claims losses settled, $S(y ; k)$}

The claims loss amount, for claims occurrence year $y$, that is settled by development year $k$, i.e., in or before development year $k$. 
It should be clear that

$$
S(y ; k)=\sum_{i=0}^{k} Z(y ; i) \text {. }
$$

From (1) follows that

$$
Z(y ; 0)=S(y ; 0)
$$

and

$$
Z(y ; k)=S(y ; k)-S(y ; k-1)
$$

and the ultimate claims loss for claims occurrence year $y$ is

$$
S(y ; n)=\sum_{i=0}^{n} Z(y ; i)
$$

The cumulative claims loss settlement information for an insurer can be represented as in Fig. 2 [2].

In Fig. 2 the $S(y ; k)$ values along the blue diagonal line represent the claims loss amounts, for claims occurrence years $Y-n$ to $Y$, that were settled up to and including the most recent completed calendar year, $Y$ [2].

\section{DEVELOPMENT FACTORS}

The use of run-off triangles for estimating future claims loss payments requires the assumption that the development of settled claims losses follows the same pattern for every claims occurrence year (see [2], [7]). Furthermore, it is assumed that this pattern will hold for future payments, i.e., the pattern will continue into the future.

Development pattern for incremental claims losses settled, $\vartheta$

The parameter vector $(\vartheta(0), \vartheta(1), \ldots, \vartheta(n))$, with $\sum_{k=0}^{n} \vartheta(k)=1$, where, for any claims occurrence year $y$ and for any development year $k \in\{0 ; 1 ; \ldots ; n\}$,

$$
\vartheta(k)=\frac{E[Z(y ; k)]}{E[S(y ; n)]} .
$$

Implicit in (2) is the assumption that, for any claims development year $k, \frac{E[Z(y ; k)]}{E[S(y ; n)]}$ is identical for every claims occurrence year $y$.

Development pattern for cumulative claims losses settled, $\gamma$

The parameter vector $(\gamma(0), \gamma(1), \ldots, \gamma(n))$, with $\gamma(n)=1$, where, for any claims occurrence year $y$ and for any development year $k \in\{0 ; 1 ; \ldots ; n\}$,

$$
\gamma(k)=\frac{E[S(y ; k)]}{E[S(y ; n)]} .
$$

Implicit in (3) is the assumption that, for any claims development year $k, \frac{E[S(y ; k)]}{E[S(y ; n)]}$ is identical for every claims occurrence year $y$ [7].
Development pattern for cumulative claims loss settlement factors, $\varphi$

The parameter vector $(\varphi(0), \varphi(1), \ldots, \varphi(n))$, where, for any claims occurrence year $y$ and for any development year $k \in\{0 ; 1 ; \ldots ; n\}$

$$
\varphi(k)=\frac{E[S(y ; k)]}{E[S(y ; k-1)]} .
$$

Implicit in (4) is the assumption that, for any claims development year $k \geq 1, \frac{E[S(y ; k)]}{E[S(y ; k-1)]}$ is identical for every claims occurrence year $y$.

\section{Estimation Using The CHAin-LAdDER MeTHOD}

The chain-ladder method (CLM) is a simple method for estimating future claims loss settlement amounts. This run-off triangle technique uses only the $\varphi(k)$ parameters and, therefore, explicitly uses (4). Other run-off triangle techniques such as the Bornhuetter-Ferguson method require the use of prior estimators of the ultimate claims losses and prior estimators of the $\gamma(k)$ parameters. These prior estimators may be based on information contained in the run-off triangle itself or on information obtained from external observations or experience (see [7]). The use of appropriate prior estimators may improve the reliability of the estimations. The choice of appropriate prior estimators required for other run-off triangle techniques is an important actuarial decision and is not considered in this paper.

In order to estimate the $Z(y ; k)$ for those cells in the runoff triangle where $y+k>Y$ (i.e. the incremental claims loss amounts that will be settled in the future) the CLM is used to estimate firstly the claims loss settlement factors for every $k \in\{0 ; 1 ; \ldots ; n\}$. These factors are then used to derive the estimators of the $S(y ; k)$ and, ultimately, the $Z(y ; k)$.

CLM estimator for $\varphi(k), \varphi^{C L}(k)$

The estimator for $\varphi(k)$ derived by using the CLM.

CLM estimator for $S(y ; k), S^{C L}(y ; k)$

The estimator for $S(y ; k)$ derived by using the CLM.

CLM estimator for $Z(y ; k), Z^{C L}(y ; k)$

The estimator for $Z(y ; k)$ derived by using the CLM.

The CLM estimator for $\varphi(k)$ is

$$
\varphi^{C L}(k)=\frac{\sum_{y=Y-n}^{Y-k} S(y ; k-1)}{\sum_{y=Y-n}^{Y-k} S(y ; k)}
$$

for any $k \in\{0 ; 1 ; \ldots ; n\}$. 
The CLM estimator for $S(y ; k)$, where $y+k>Y$, is

$$
\begin{array}{r}
S^{C L}(y ; k)=S(y ; Y-y) \cdot \varphi^{C L}(Y-y+1) . \\
\cdot \varphi^{C L}(Y-y+2) \cdot \ldots \cdot \varphi^{C L}(k)
\end{array}
$$

Formula (6) is equivalent to

$$
S^{C L}(y ; k)=S(y ; Y-y) \cdot \prod_{j=Y-y+1}^{k} \varphi^{C L}(j)
$$

It can also be derived (and is intuitively obvious) that

$$
S^{C L}(y ; k)=S^{C L}(y ; k-1) \cdot \varphi^{C L}(k) .
$$

Figure 3 demonstrates how elements of the observed historical data are used for determining the CLM estimators for the cumulative claims loss factors, $\varphi^{C L}(k)$ using (5).

Figure 4 demonstrates how the derived $\varphi^{C L}(k)$ factors are used together with the $S(y ; Y-y)$ values along the diagonal (the most up-to-date cumulative claims settlement data) to

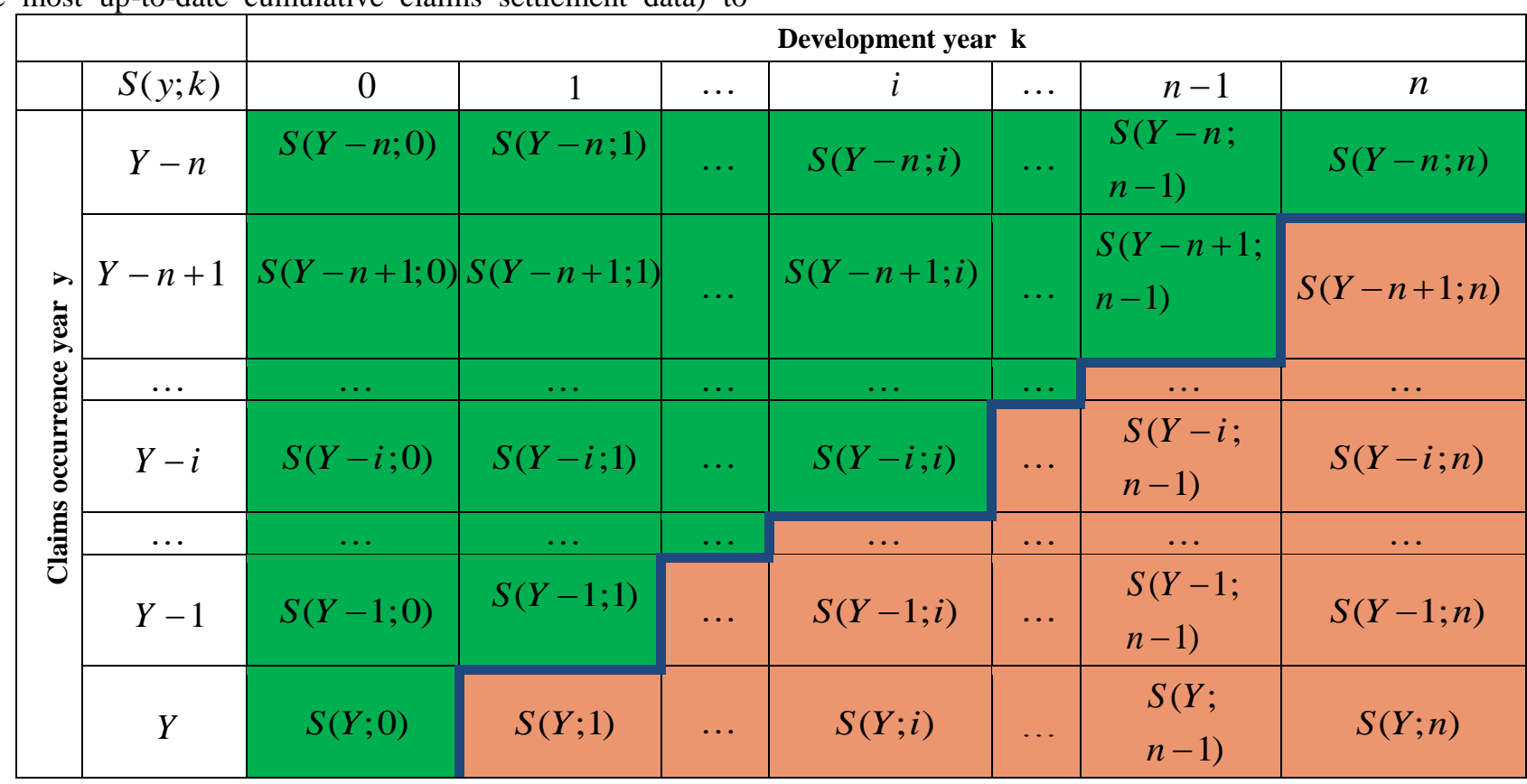

Fig. 2. Cumulative claims loss settlement data represented as a run-off triangle. calculate the estimated future cumulative claims settlement amounts using (6) and (7).

The CLM estimator for $Z(y ; k)$ is then simply calculated as

$$
Z^{C L}(y ; k)=S^{C L}(y ; k)-S^{C L}(y ; k-1)
$$

Formula (9) is based on the relationship between incremental and cumulative claims losses settled as demonstrated in (1).

Once $Z^{C L}(y ; k)$ are derived these estimated future incremental claims loss settlement amounts can be grouped by the expected year of settlement. These projected cash flows can then be discounted appropriately in order to determine the required technical provisions for these liabilities [5]. Fig. 5 demonstrates how the estimated incremental claims loss settlements can be allocated to future calendar years. 


\begin{tabular}{|c|c|c|c|c|c|c|c|c|c|}
\hline & & \multicolumn{8}{|c|}{ Development year $\mathbf{k}$} \\
\hline & $S(y ; k)$ & 0 & 1 & $\ldots$ & $i-1$ & $i$ & $\ldots$ & $n-1$ & $n$ \\
\hline \multirow{3}{*}{ 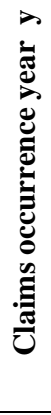 } & $Y-n$ & $S(Y-n ; 0)$ & $S(Y-n ; 1)$ & $\cdots$ & $S(Y-n ; i-1)$ & $S(Y-n ; i)$ & $\cdots$ & $\begin{array}{l}S(Y-n \\
n-1)\end{array}$ & $S(Y-n ; n)$ \\
\hline & $Y-n+1$ & $\begin{array}{l}S(Y- \\
n+1 ; 0)\end{array}$ & $\begin{array}{l}S(Y- \\
n+1 ; 1)\end{array}$ & $\cdots$ & $\begin{array}{l}S(Y-n+1 \\
i-1)\end{array}$ & $\begin{array}{l}S(Y- \\
n+1 ; i)\end{array}$ & $\cdots$ & $\begin{array}{l}S(Y-n+1 \\
n-1)\end{array}$ & \\
\hline & $\ldots$ & $\cdots$ & $\cdots$ & $\ldots$ & $\ldots$ & $\cdots$ & $\ldots$ & & \\
\hline \multirow{5}{*}{ 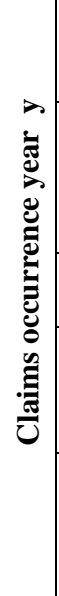 } & $Y-i$ & $S(Y-i ; 0)$ & $S(Y-i ; 1)$ & $\cdots$ & $S\left(Y-i_{1}^{\mid} i-1\right)$ & $S(Y-i ; i)$ & & & \\
\hline & $Y-i+1$ & $\begin{array}{l}S(Y- \\
i+1 ; 0)\end{array}$ & $\begin{array}{l}S(Y- \\
i+1 ; 1)\end{array}$ & $\cdots$ & $\begin{array}{l}S(Y-i+1 \\
i-1)\end{array}$ & & -- & $\sum_{y=Y-n}^{Y-i} S(y ; i)$ & \\
\hline & $\ldots$ & $\ldots$ & $\ldots$ & $\ldots$ & --- & --- & -- & $\sum_{v=Y-n}^{Y-1} S(y ; i-1)$ & \\
\hline & $Y-1$ & $S(Y-1 ; 0)$ & $S(Y-1 ; 1)$ & & \multirow{2}{*}{\multicolumn{3}{|c|}{$\varphi^{C L}(i)=\frac{\sum_{y=Y-n} S(y ; i-1)}{\sum_{y=Y-n}^{Y-i} S(y ; i)}$}} & & \\
\hline & $Y$ & $S(Y ; 0)$ & & & & & & & \\
\hline
\end{tabular}

Fig. 3. Determining the CLM estimator for the cumulative claims loss settlement factor, $\varphi^{C L}(k)$ using run-off triangle information.

\begin{tabular}{|c|c|c|c|c|c|c|c|c|}
\hline \multirow{2}{*}{\multicolumn{2}{|c|}{$\begin{array}{c}S(y ; k), \\
S^{C L}(y ; k)\end{array}$}} & \multicolumn{7}{|c|}{ Development year $\mathbf{k}$} \\
\hline & & 0 & 1 & & $i$ & & $n-1$ & $n$ \\
\hline \multirow{4}{*}{ 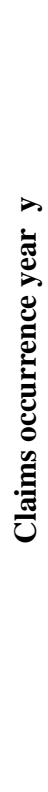 } & $Y-n$ & $S(Y-n ; 0)$ & $S(Y-n ; 1)$ & $\ldots$ & $S(Y-n ; i)$ & $\ldots$ & $\begin{array}{l}S(Y-n \\
n-1)\end{array}$ & $S(Y-n ; n)$ \\
\hline & $Y-n+1$ & $\begin{array}{l}S(Y- \\
n+1 ; 0)\end{array}$ & $\begin{array}{l}S(Y- \\
n+1 ; 1)\end{array}$ & $\ldots$ & $S(Y-n+1 ; i)$ & $\ldots$ & $\begin{array}{c}S(Y-n+1 \\
n-1)\end{array}$ & $\begin{array}{c}S^{C L}(Y- \\
n+1 ; n)= \\
S(Y-n+1 \\
n-1) \\
\cdot \varphi^{C L}(n)\end{array}$ \\
\hline & $\ldots$ & $\ldots$ & $\ldots$ & $\ldots$ & $\ldots$ & $\ldots$ & $\ldots$ & .. \\
\hline & $Y-i$ & $S(Y-i ; 0)$ & $S(Y-i ; 1)$ & $\ldots$ & $S(Y-i ; i)$ & $\cdots$ & $\cdots$ & $\begin{array}{l}S^{C L}(Y-i ; n) \\
=S(Y-i ; i) \\
\cdot \prod^{n} \varphi^{C L}(j)\end{array}$ \\
\hline
\end{tabular}




\begin{tabular}{|c|c|c|c|c|c|c|c|}
\hline$Y-i+1$ & $\begin{array}{l}S(Y- \\
i+1 ; 0)\end{array}$ & $\begin{array}{l}S(Y- \\
i+1 ; 1)\end{array}$ & $\ldots$ & $\begin{array}{c}S^{C L}(Y- \\
i+1 ; i)= \\
S(Y-i+1 ; i-1) \\
\cdot \varphi^{C L}(i)\end{array}$ & $\ldots$ & & $\begin{array}{c}S^{C L}(Y- \\
i+1 ; n)= \\
S(Y-i+1 ; i-1) \\
\cdot \prod_{j=1}^{n} \varphi^{C L}(j)\end{array}$ \\
\hline$\ldots$ & $\ldots$ & $\ldots$ & $\ldots$ & $\ldots$ & $\ldots$ & $\ldots$ & $\ldots$ \\
\hline$Y-1$ & $S(Y-1 ; 0)$ & $S(Y-1 ; 1)$ & $\ldots$ & $\begin{array}{c}S^{C L}(Y-1 ; i) \\
=S(Y-1 ; 1) \\
\cdot \varphi^{C L}(2) \cdot \varphi^{C L}(3) \\
\cdot \ldots \cdot \varphi^{C L}(i)\end{array}$ & $\ldots$ & $\ldots$ & $\begin{array}{l}S^{C L}(Y-1 ; n) \\
=S(Y-1 ; 1) \\
\cdot \prod_{j=2}^{n} \varphi^{C L}(j)\end{array}$ \\
\hline$Y$ & $S(Y ; 0)$ & $\begin{array}{l}S^{C L}(Y ; 1) \\
=S(Y ; 0) \\
\cdot \varphi^{C L}(1)\end{array}$ & & $\begin{array}{c}S^{C L}(Y ; i) \\
=S(Y ; 0) \\
\cdot \varphi^{C L}(1) \cdot \varphi^{C L}(2) \\
\cdot \ldots \cdot \varphi^{C L}(i)\end{array}$ & & $\begin{array}{c}S^{C L}(Y ; n-1) \\
=S(Y ; 0) \\
\cdot \prod_{j=1}^{n-1} \varphi^{C L}(j)\end{array}$ & $\begin{array}{l}S^{C L}(Y ; n) \\
=S(Y ; 0) \\
\cdot \prod_{j=1}^{n} \varphi^{C L}(j)\end{array}$ \\
\hline
\end{tabular}

Fig. 4. Determining the CLM estimators for the future cumulative claims loss settlement amounts, $S^{C L}(y ; k)$.

\begin{tabular}{|c|c|c|c|c|c|c|c|c|}
\hline \multirow{2}{*}{\multicolumn{2}{|c|}{$S^{C L}(y ; k)$}} & \multicolumn{7}{|c|}{ Development year } \\
\hline & & 0 & 1 & $\ldots$ & $i$ & $\ldots$ & $n-1$ & $n$ \\
\hline \multirow{8}{*}{ 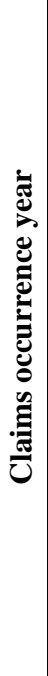 } & $Y-n$ & & & & & & & \\
\hline & $Y-n+1$ & & & & & & & $S^{C L}(Y-n+1 ; n)$ \\
\hline & $\ldots$ & & & & & & $\ldots$ & $\ldots$ \\
\hline & $Y-i$ & & & & & & $S^{C L}(Y-i ; n-1)$ & $S^{C L}(Y-i ; n)$ \\
\hline & $Y-i+1$ & & & & $S^{C L}(Y-i+1 ; i)$ & & $S^{C L}(Y-i+1 ; n-1)$ & $S^{C L}(Y-i+1 ; n)$ \\
\hline & $\ldots$ & & & & $\ldots$ & $\ldots$ & $\ldots$ & $\ldots$ \\
\hline & $Y-1$ & & & $\cdots$ & $S^{C L}(Y-1 ; i)$ & $\ldots$ & $S^{C L}(Y-1 ; n-1)$ & $S^{C L}(Y-1 ; n)$ \\
\hline & $Y$ & & $S^{C L}(Y ; 1)$ & & $S^{C L}(Y ; i)$ & & $S^{C L}(Y ; n-1)$ & $S^{C L}(Y ; n)$ \\
\hline
\end{tabular}




\begin{tabular}{|c|c|}
\hline Calendar year & Estimated claims loss settlement amounts \\
\hline$Y+1$ & $S^{C L}(Y ; 1)+S^{C L}(Y-1 ; 2)+\ldots+S^{C L}(Y-n+1 ; n)$ \\
\hline$\ldots$ & $\ldots$ \\
\hline$Y-i+n$ & $S^{C L}(Y ; n-i)+S^{C L}(Y-1 ; n-i+1)+\ldots+S^{C L}(Y-i ; n)$ \\
\hline$Y-i+n+1$ & $S^{C L}(Y ; n-i+1)+S^{C L}(Y-1 ; n-i+2)+\ldots+S^{C L}(Y-i+1 ; n)$ \\
\hline$\ldots$ & $\ldots$ \\
\hline$Y-1+n$ & $S^{C L}(Y ; n-1)+S^{C L}(Y-1 ; n)$ \\
\hline$Y+n$ & $S^{C L}(Y ; n)$ \\
\hline
\end{tabular}

Fig. 5. Allocating estimated future claims loss settlements to future calendar years.

In its usual form the chain ladder algorithm suggests that total claims for the current year are constructed in a similar way to their claims on the previous year. The algorithm evaluates development factors as the ratio of total sum of claims this year to the amount of total claims in the same period of the previous year. Thus, the estimate of development factor for the $j$-th column of the table in Figure 3 is equal to (10).

$$
\hat{\lambda}_{j}=\frac{\sum_{i=1}^{t-j+1} S_{i j}}{\sum_{i=1}^{t-j+1} S_{i, j-1}} .
$$

Equation (10) is based on the following model:

$E\left(S_{i j} \mid S_{i 1}, S_{i 2}, \cdots, S_{i, j-1}\right)=\lambda_{j} S_{i, j-1}, \quad j=2, \ldots, t$.

and (10) is the estimate of $\lambda_{j}$. Advantage of the method is of standard calculations, but there is no clear idea of how to estimate its properties in details.

The expected final total loss $E\left(S_{i j}\right)$ is estimated by multiplying the latest loss $S_{i, t-i+1}$ to vector $\lambda$, the estimation is equal (12).

$$
E\left(S_{i t}\right)=\left(\prod_{j=t-i+2}^{t} \hat{\lambda}_{j}\right) S_{i, t-i+1} .
$$

\section{RELATED WORKS}

Instead of using traditional actuarial methods to complete an IBNR run-of triangle can be described by one generalized linear model. In Fig.1, the random variables $Z_{i j}$ for $i, j=0,1, \ldots, n$ denote the claim figure for year of origin $i$ and year of development $j$, meaning that the claims were paid in calendar year $i+j-1$. For $(i, j)$ combinations with $i+j-1<n, Z_{i j}$ has already been observed, otherwise it is a future observation. As well as claims actually paid, this figure may also be used to denote loss ratios quantities. We take a multiplicative model, with a parameter for each row $i$, each column $j$ and each diagonal $\mathrm{k}=i+j-1$, as follows:

$$
Z_{i j}=\alpha_{i} \cdot \beta_{j} \cdot \gamma_{k}
$$

The deviation of the observation on the left hand side from its mean value on the right hand side is attributed to chance. As one sees, if we assume further that the random variables $Z_{i j}$ are independent and restrict their distribution to be in the exponential dispersion family, (13) is a generalized linear model [3]. Year of origin $i$, year of development $j$ and calendar year $k=i+j-1$ act as explanatory variables for the observation $Z_{i j}$. The expected value of $Z_{i j}$ is the exponent of the linear form

$$
\log \alpha_{i}+\log \beta_{j}+\log \gamma_{k} .
$$

Note that the covariates are all dummies representing group membership for rows, columns and diagonals in Fig.1. We will determine maximum likelihood estimates of

the parameters $\alpha_{i}, \beta_{j}$ and $\gamma_{k}$, under various assumptions for the probability distribution of the $Z_{i j}$. It will turn out that in this simple way, we can generate quite a few widely used IBNR techniques. Having found estimates of the parameters, it is easy to extend the triangle to a square, simply by taking

$$
\hat{Z}_{i j} \approx \hat{\alpha}_{i} \cdot \hat{\beta}_{j} \cdot \hat{\gamma}_{k}
$$

A problem is that we have no data on the values of the $\gamma_{k}$ for future calendar years $k$ with $k>n$. The problem can be solved, for example, by assuming that the $\gamma_{k}$ have a geometric distribution, for some real number $\gamma$.

Kramer [4] shows that if the estimation $\hat{Z}_{i j}$ received through parameter substitutions to their average values, the result is very similar to that obtained by the chain ladder method. The resulting estimation $\hat{Z}_{i j}$ is not maximum likelihood estimation, but it serves to illustrate the similarity chain method with the generalized linear model method [3].

Moreover, if all the geometric means are replaced by the arithmetic averages, the estimators of parameters of the both models are equivalent. Thus, two methods of estimation, the chain method and generalized linear model, will produce the same results. The structure of the models is identical, and the only difference is the technique of estimation. It can be argued that the evaluation of linear models is the best in a statistical sense, but it should also be emphasized that by using the linear model instead of the chain method, there are no radical changes. 


\section{CONCLUSIONS}

This paper examines the various models that are available in the linear model of the chain ladder method. It shows that the actuary of an insurance company will find all these models useful. The following points are important. The standard error of a linear generalized model is very important. You must set the most likely actuary «top» reserve for the entire company on this type of insurance, rather than for each triangle. This should reduce the relative size of the standard error of even more. There are several statistical methods that are valid for the evaluation of the claims; they all have partial advantages over the classical method of estimation - method of chain ladder. A generalized linear model is a big step forward from the chain ladder method and technology opens the way to more complex methods of estimation.

\section{REFERENCES}

[1] Brosemer, M. (2011): Treatment of Technical provisions under solvency, Munich Re, Solvency Consulting Knowledge Series, p.1-8.

[2] GDV (Gesamtverband der Deutschen Versicherungswirtschaft e. V.) (2011): Methoden zur Schätzung von Schaden- und Prämienrückstellungen in der Kompositversicherung Überarbeitete Fassung.http://www.gdv.de/wpcontent/uploads/2011/11/Broschuere_Schaden_und_Praemienrueckste llungen_in_der_Kompositversicherung_2011.pdf

[3] Kaas, R., Goovaerts, M., Dhaene, J., Deniut, M. (2001): Modern Actuarial Risk Theory. Kluwer Academic Publishers, Dordrecht

[4] Kremer (1982): IBNR-Claims and the Two-Way Model of ANOVA. Scand.Act.J., Vol.1, p.47-55.

[5] Norberg, R. (1993): Prediction of Outstanding Liabilities in Non-Life Insurance, ASTIN Bulletin, Vol 23, No 1, p. 95-115. http://dx.doi.org/10.2143/AST.23.1.2005103

[6] Schiegl, M. (2002): On the Safety Loading for Chain Ladder Estimates: A Monte Carlo Simulation Study, ASTIN Bulletin, Vol. 32, No. 107.

[7] Schmidt, K. (2006): Methods and Models of Loss Reserving Based on Run-off Triangles: A Unifying Survey. In: Casualty Actuarial Society Forum Fall 2006, 269 - 317.

[8] Schmidt, K. (2008): Bornhuetter-Ferguson as a General Principle of Loss Reserving, ASTIN Manchester, July 14-16.
[9] Quarg, G. (2004): Munich Chain Ladder, Blätter der Deutschen Gesellschaft für Versicherungs- und Finanzmathematik, Vol. 26, No. 4, 2004, p. 597-630.

[10] Verrall, R. (1991): Statistical Methods for the Chain Ladder Technique, ASTIN Bulletin, Vol. 20, No. 2, p. 393-446.

Aleksandrs Matvejevs, email: Aleksandrs.Matvejevs@rtu.lv, has graduated from Riga Technical University, the Faculty of Automation and Computing Technique. He received the Doctoral Degree in 1991 and became an Associate Professor at Riga Technical University in 1999. Aleksandrs Matvejevs is a Doctor of Mathematics (speciality, including number theory with applications, mathematical modeling and parameter estimation with the method of associative modeling). $\mathrm{He}$ is the author of about 30 scientific publications and conference papers.

Anatoliy Malyarenko, email: Anatoliy.Malyarenko@mdh.se, graduated with distinction from Kiev State University, Ukraine, in 1979. In 1985, he defended the Doctoral Thesis. He has worked at Kiev State University since 1981, and at the International Mathematical Centre of the National Academy of Sciences of Ukraine since 1992. Since 2000 he has been working at Mälardalen University, Västerås, Sweden, currently as a Full Professor at the Division of Applied Mathematics.

His publications include 1 book, 2 edited books, 29 international peerreviewed papers, 9 papers in books and peer-reviewed proceedings of conferences, 8 research reports, and 26 other publications. Current research interests include the spectral theory of multidimensional random functions and development of programming systems for financial and actuarial mathematics.

Andrejs Matvejevs, email: Andrejs.Matvejevs@rtu.lv, has graduated from Riga Technical University, the Faculty of Automation and Computing Technique. He received the Doctoral Degree in 1989 and became an Associate Professor at Riga Technical University in 2000 and a Full Professor in 2005. He has made the most significant contribution to the field of actuarial mathematics. Andrejs Matvejevs is a Doctor of Technical Sciences in Information Systems. Until 2009 he was a Chief Actuary at the insurance company "BALVA". For more than 25 years he has taught at Riga Technical University and Riga International College of Business Administration, Latvia. His previous research was devoted to solving dynamical systems with random perturbation. His current professional research interests include applications of Markov chains to actuarial technologies: mathematics of finance and security portfolio.

$\mathrm{He}$ is the author of about 40 scientific publications, two textbooks and numerous conference papers. 